\title{
The effect of laser revascularization of the liver on portal hemodynamics in cirrhotic patients: preliminary study
}

\begin{abstract}
Background and aims: Portal hypertension leads to serious complications such as variceal bleeding and is responsible for significant morbidity and mortality in cirrhotic patients. The aim of this research was to study the influence of improving of the microenvironment within the liver through formation of new vessels and extracellular matrix remodelling using a high-intensity laser radiation on portal hemodynamics in cirrhotic patients
\end{abstract}

Patients and methods: The laser revascularization of the liver was carried out on 20 cirrhotic patients with portal hypertension. A diode laser SHARPLAN-6020 (Israel) was used as a source of radiation. Observation of portal hemodynamics prior to and following surgical intervention was carried out using impulse Doppler. The diameter of the portal vein and diameter of the splenic vein was determined as well as their maximal flow velocity and flow volume. Based on this data the congestion index of the portal vein velocity was calculated.

Results: In the first 10 days after the laser revascularization of the liver there was a considerable increase in maximal flow velocity of the portal vein $(p<0.05)$, and while flow volume of the portal $\mathrm{v}$ ein practically did not change because of a certain decrease in diameter of the portal vein $(\mathrm{p}>0.05)$, this contributed to a marked reduction in congestion index of the portal vein velocity $(\mathrm{p}<0.05)$. There was a subsequent decline in maximal flow velocity of the portal vein and by the end of the year it was at initial values. Other indices of portal blood flow (diameter of the portal vein, flow volume of the portal vein and congestion index of the portal vein velocity) showed a downward trend and a year later they were positively lower than the initial values $(\mathrm{p}<0.05)$.

Conclusion: Improving of the microenvironment within the liver through formation of new vessels and extracellular matrix remodelling using a high-intensity laser radiation may be a promising method of treating both liver cirrhosis and its complications.

Keywords: liver cirrhosis, portal hypertension, high-intensity laser radiation, laser revascularization of the liver, portal hemodynamics
Volume 2 Issue 5 - 2015

\section{Dmitry Victorovich Garbuzenko, Victor Nikolaevich Bordunovskii \\ Department of Faculty Surgery, South Ural State Medical University, Russia}

Correspondence: Dmitry Victorovich Garbuzenko, Professor of Department of Faculty Surgery, South Ural State Medical University, Box 12317, Chelyabinsk, 454080, Russia, Tel +8-9097459826, Fax +8-35I-2687772, Email garb@inbox.ru

Received: August 05, 2015 | Published: October 13, 2015
Abbreviations: ØРV, diameter of the portal vein; VPV, maximal flow velocity of the portal vein; WPV, flow volume of the portal vein; $Ø \mathrm{SV}$, diameter of the splenic vein; VSV, maximal flow velocity of the splenic vein; WSV, flow volume of the splenic vein; $\mathrm{CI}$, congestion index of the portal vein velocity

\section{Introduction}

Portal hypertension leads to serious complications such as variceal bleeding and is responsible for significant morbidity and mortality in cirrhotic patients. At the base of the pathogenesis of portal hypertension is the increase in hepatic vascular resistance to portal blood flow. It is now established that the reason for this, in addition to gross structural changes in the liver due to diffuse fibrosis and the formation of nodules of regenerating hepatocytes, remodeling and capillarization of the hepatic sinusoids, is endothelial dysfunction and the disorder of paracrine interactions between damaged hepatocytes, sinusoidal endothelial cells, Kupffer cells and activated hepatic stellate cells of the liver. ${ }^{1}$ Further development of splanchnic hyperemia, the formation of collateral circulation and established hyper dynamic circulation, as a result of complex processes of angiogenesis, vascular remodeling and endothelial dysfunction, contributes to the progression of portal hypertension. ${ }^{2}$
We hypothesized that one of the methods of correction of disturbances of portal hemodynamics under liver cirrhosis can be improving of the microenvironment within the liver through formation of new vessels and extracellular matrix remodelling, in particular, using a high-intensity laser radiation. Earlier it was determined that when the cirrhotic liver is exposed to high-intensity infrared laser radiation $(\lambda$ - 1064 and $805 \mathrm{~nm}$ ) the zone of destruction sees increased expression of vascular endothelial growth factor and the basic fibroblasts growth factor, as well as higher activity of matrix metalloproteinases and proteases of the plasmin system, which stimulates the formation of new vessels. ${ }^{3,4}$ In the preliminary experimental study on rabbits with the model of CCL4-induced liver cirrhosis by means of the application of histological, morphometric and electron microscope methods we conducted an integrated assessment of the processes of alteration, compensation and adaptation after exposing the liver to high-intensity laser radiation, using a diode laser $(\lambda-810 \mathrm{~nm})$. As a result it was noted that against the background of partial involution of fibrous tissue in the animals' livers there were signs of reparative regeneration with marked neoangiogenesis, which prevailed in the areas exposed to the laser beam. ${ }^{5}$ The aim of this research was to study the influence of improving of the microenvironment within the liver through formation of new vessels and extracellular matrix remodelling using a high-intensity laser radiation on portal hemodynamics in cirrhotic patients. 


\section{Patients and methods}

A total of 20 consecutive patients with biopsy confirmed cirrhosis who were admitted to Clinic of Department of Faculty Surgery South Ural State Medical University were evaluated. Each of them had been hospitalized for variceal bleeding and received octreotide and terlipressin during the acute episode. The etiology of cirrhosis was alcohol-induced in 6 patients, chronic viral hepatitis in 7 patients and primary biliary cirrhosis in 2 patients and cryptogenous in 5 patients. There were 10 male and 10 female patients with a mean age of 52.8 \pm 2.79 (20-68) years. Patients without severe hepatic failure, ascites, congestive heart failure, ultrasonographic data suggesting about a hepatocellular carcinoma, portal thrombosis and a dilated paraumbilical veins, as well as which was tolerant to the action of nonselective $\beta$-adrenergic blockers were included from study. The study was conducted according to the principles of Declaration of Helsinki. Informed consent was obtained from all patients and the study was approved by the local ethics committee. Detailed disease history was obtained from all patients and physical examination was performed. Hematological and biochemical work-up included measurement of hemoglobin, total leukocyte count, platelet count, serum concentrations of bilirubin (total and conjugated), protein, albumin, alanine aminotransferase and aspartate aminotrasferase. Furthermore, the hemostatic factors of their peripheral blood, such as prothrombin time, International Normalized Ratio, activated partial thromboplastin time, activated recalcification time, soluble fibrin-monomer complexes, fibrinogen, Hageman factor dependent fibrinolysis, were examined. All patients were tested for HBV-DNA, $\mathrm{HBeAg}, \mathrm{HBsAg}$, anti-HBcAg, antibodies to hepatitis $\mathrm{C}$ virus, and antimitochondrial antibodies to determine the cause of liver cirrhosis. For each patient, a modified Child-Pugh score was calculated. Four of them had Child-Pugh A and sixteen had Child-Pugh B liver cirrhosis.

All patients underwent esophagogastroduodenoscopy for assessment of esophageal varices, the degree of which was defined according to the World Gastroenterology Organisation Global Guidelines (January, 2014). Herewith, large esophageal varices (> 5 $\mathrm{mm}$; occupying more than one-third of the esophageal lumen) were identified in 14 patients. All patients underwent ultrasonography and the following details were recorded: maximum vertical span of the liver, nodularity of liver surface; spleen size (length of its longest axis); diameter of the portal and splenic veins; presence of portosystemic collaterals, ascites, a hepatocellular carcinoma and portal thrombosis.

\section{Hemodynamic studies}

Portal hemodynamics was evaluated by Doppler ultrasonography, which is an important as a supplementary tool for the assessment of portal hypertension and response to treatment, when investigation the hepatic venous pressure gradient is not feasible. ${ }^{6}$ Hemodynamic studies were performed on 7-10 day after admission, when the gastrointestinal bleeding had ceased and hemodynamic conditions had stabilized, as well as on day 10, after 3 months and 1 year following laser revascularization of the liver. Blood flow parameters were measured at $8 \mathrm{am}$ after overnight fasting. Investigation was carried out in real time by means of colored scanner "Acuson-ASPEN" (USA) by $3.5 \mathrm{mHz}$ convex sensor using impulse Doppler, with the patients lying supine. The diameter, maximal flow velocity and flow volume of the portal vein and splenic veins were measured. Portal and splenic vein diameter was measured from inner side to inner side at the point where peak flow velocities were obtained.

Based on this data the congestion index of the portal vein velocity was calculated:

$$
\text { Congestion index of the portal vein velocity }=\frac{\text { cross }- \text { section area portal vein }}{\text { maximal flow velocity portal vein }}
$$

A number of publishing have shown that flow volume of the splenic vein and congestion index are independent risk factors for variceal bleeding, ${ }^{7}$ moreover, congestion index correlates with values of the hepatic venous pressure gradient. ${ }^{8}$ Control study of portal hemodynamics was performed in 17 healthy volunteers.

\section{Laser revascularization of the cirrhotic liver}

The laser revascularization of the cirrhotic liver was developed in Department of Faculty Surgery South Ural State Medical University (Russian Federation invention patent № 2186545 dated 10 August, 2002). Preoperative preparation was no different from the common one when operating on abdominal cavity organs. A diode laser SHARPLAN-6020 (Israel) was used as a source of radiation. The laparoscopic surgery was performed under general anaesthesia with the patient lying supine. The abdominal cavity puncture to create pneumoperitoneum (10-12 $\mathrm{mm}$ of mercury) and insertion of the first trocar was done along the center line between the navel and the midway point from the navel to the xiphisternum taking into account the physique of patient. This point is optimal for viewing of the entire diaphragmal surface of the liver by laparoscope $90^{\circ}$. When necessary, partial mobilization of the liver with the intersection of the falciform, coronary and triangular ligaments was performed. The inspection of its visceral surface was carried out after lifting the right and/or left lobe and moving the epiploon to the Fowler position. The insertion point of trocars for positioning the flexifiber quartz light guide of $\overleftarrow{\varnothing} 0.6 \mathrm{~mm}$ was chosen individually in the right and left hypochondrium based on the size of the liver and the nature of the pathologic process. A laser beam of $\lambda-810 \mathrm{~nm}, \mathrm{P}=5-10 \mathrm{~W}$ (the power was adjusted individually depending on the liver tissue density) was supplied through the light guide, the mode used was pulse with impulse duration of $0.1 \mathrm{sec}$, pause $-0.25 \mathrm{sec}$, exposure time - 10-14 sec), to create 10-20 channels up to 2-3 cm deep (one segment after another) on the diaphragmal surface of each liver lobe based on the size and structure of the organ (Figure 1). The laser was also used to coagulate separate bleeding sites of the parenchyma. The duration of the surgery was under 20-30 min. There were no intraoperative complications.

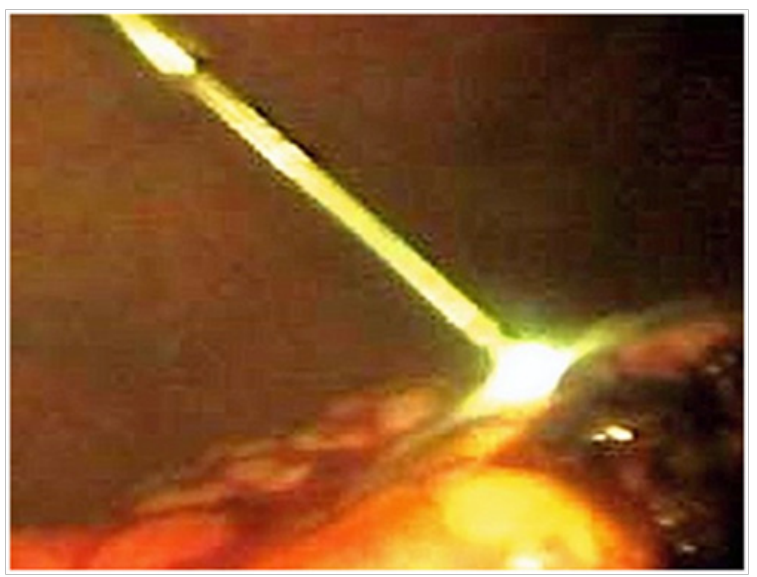

Figure I Laser revascularization of the cirrhotic liver.

\section{Statistical analysis}

Statistical calculations were made using SPSS for the windows computer program. There were calculated values $\mathrm{M}$, their standard deviation (m) and a $95 \%$ confidence interval, and used non- 
parametric Wilcoxon rank-sum test. Data obtained at the end of the study was expressed as mean $\pm \mathrm{SD}$. The differences were considered to be accurate at $\mathrm{p}<0.05$.

\section{Results and discussion}

Liver cirrhosis causes important hemodynamic changes. Despite the increase the inflow of splanchnic blood, portal blood flow to the liver decreases because of increasing sinusoidal resistance and development of portosystemic collateral vessels. These hemodynamic changes influence the degree of portal hypertension and liver dysfunction. Thereby, it is necessary to reduce the inflow of splanchnic blood to the portal vein, while maintaining portal circulation, which participates in hepatic perfusion. ${ }^{9}$ Indicators of portal hemodynamics in cirrhosis patients in our study corresponded to these changes (Table 1). In the first 10 days after the laser revascularization of the liver there was a considerable increase in maximal flow velocity of the portal vein $(p<0.05)$, and while flow volume of the portal vein practically did not change because of a certain decrease in diameter of the portal vein $(\mathrm{p}>0.05)$, this contributed to a marked reduction in congestion index of the portal vein velocity $(\mathrm{p}<0.05)$. There was a subsequent decline in maximal flow velocity of the portal vein and by the end of the year it was at initial values. Other indices of portal blood flow (diameter of the portal vein, flow volume of the portal vein and congestion index of the portal vein velocity) showed a downward trend and a year later they were positively lower than the initial values $(\mathrm{p}<0.05)$. Thus, diameter of the portal vein decreased by $19.8 \%$, flow volume of the portal vein by $38.5 \%$, and congestion index of the portal vein velocity by $36.4 \%$, which indicates lower congestion in the portal venous system. As a consequence, the attributes of splenic venous blood flow were also improved. Starting from the 10th day after the operation, diameter of the portal vein, maximal flow velocity of the splenic vein and flow volume of the splenic vein showed a downward trend $(p>0.05)$. Despite the fact that a year later diameter of the splenic vein was somewhat increased $(p>0.05)$, the maximal flow velocity of the splenic vein $(\mathrm{p}>0.05)$ and flow volume of the splenic vein $(\mathrm{p}<0.05)$ indices were below the initial values. For example, the maximal flow velocity of the splenic vein values decreased by $13.4 \%$, and flow volume of the splenic vein by $38.5 \%$ (Table 2). Unfortunately, due to the small number of patients we were unable to conduct a comparative analysis of long-term clinical benefits at a sufficient level of statistical accuracy. However, it was noticeable that there was a beneficial effect of laser revascularization of the liver on the esophageal varices. Thus, varices disappeared in 3 patients, decreased in 3 others, and only 2 patients had increased varices. 4 patients had esophageal variceal bleeding after the surgery.

Table I Doppler sonographic indices of portal hemodynamics in control groups and cirrhotic patients, $M \pm m$

\begin{tabular}{lll}
\hline Index & Control groups $(\mathbf{n}=17)$ & Cirrhotic patients $(\mathbf{n}=20)$ \\
\hline$\varnothing P V(\mathrm{~mm})$ & $12.1 \pm 0.2$ & $12.6 \pm 0.8$ \\
VPV $(\mathrm{sm} / \mathrm{sec})$ & $19.1 \pm 0.1$ & $15.8 \pm 1.6^{*}$ \\
WPV $(1 / \mathrm{min})$ & $1.2 \pm 0.1$ & $1.3 \pm 0.4$ \\
$\varnothing S V(\mathrm{~mm})$ & $8.0 \pm 0.4$ & $10.1 \pm 0.8^{*}$ \\
VSV $(\mathrm{sm} / \mathrm{sec})$ & $19.3 \pm 1.5$ & $21.6 \pm 2.8$ \\
WSV $(1 / \mathrm{min})$ & $0.5 \pm 0.1$ & $1.3 \pm 0.4^{*}$ \\
$\mathrm{Cl}(\mathrm{sm} / \mathrm{sec})$ & $0.08 \pm 0.01$ & $0.11 \pm 0.02^{*}$ \\
\hline
\end{tabular}

Note: Ø̋P: Diameter of the Portal Vein; VPV: Maximal Flow Velocity of the Portal Vein; WPV: Flow Volume $O$ of the Portal Vein; ØSV: Diameter of the Splenic Vein;VSV: Maximal Flow Velocity of the Splenic Vein;WSV: Flow Volume of the Splenic Vein; Cl: Congestion Index of the Portal Vein Velocity $*$ - accuracy of differences from the control group $(p<0.05)$. Wilcoxon rank-sum test applied.

Table 2 Doppler sonographic indices of portal hemodynamics in cirrhotic patients after laser revascularization of the liver, $M \pm m$

\begin{tabular}{|c|c|c|c|c|}
\hline \multirow{2}{*}{ Index } & \multicolumn{4}{|c|}{ Examination period } \\
\hline & $0(n=20)$ & 10th day $(n=20)$ & 3 months $(n=19)$ & I year $(n=\mid 4)$ \\
\hline ØРPV (mm) & $12.6 \pm 0.8$ & $12.0 \pm 0.7$ & $12.0 \pm 0.7$ & $10.1 \pm 0.5^{*}$ \\
\hline VPV (sm/sec) & $15.8 \pm 1.6$ & $20.6 \pm 2.1 *$ & $15.1 \pm 1.0$ & $15.8 \pm 2.9$ \\
\hline WPV (I/min) & $1.3 \pm 0.4$ & $1.3 \pm 0.2$ & $0.9 \pm 0.1$ & $0.8 \pm 0.2^{*}$ \\
\hline Ø̋SV (mm) & $10.1 \pm 0.8$ & $9.4 \pm 1.0$ & $9.7 \pm 0.9$ & $10.7 \pm 0.7$ \\
\hline VSV (sm/sec) & $21.6 \pm 2.8$ & $20.9 \pm 1.9$ & $20.7 \pm 1.7$ & $18.7 \pm 1.8$ \\
\hline WSV (I/min) & $1.3 \pm 0.4$ & $1.2 \pm 0.4$ & $1.0 \pm 0.3$ & $0.8 \pm 0.2 *$ \\
\hline $\mathrm{Cl}(\mathrm{sm} / \mathrm{sec})$ & $0.11 \pm 0.02$ & $0.07 \pm 0.01 *$ & $0.09 \pm 0.01 *$ & $0.07 \pm 0.01 *$ \\
\hline
\end{tabular}

Note: ØРV: Diameter of the Portal Vein; VPV: Maximal Flow Velocity of the Portal Vein; WPV: Flow Volume of the Portal Vein; ØSV: Diameter of the Splenic Vein; VSV: Maximal Flow Velocity of the Splenic Vein; WSV: Flow Volume of the Splenic Vein; Cl: Congestion Index of the Portal Vein Velocity $*$ - accuracy of differing from the initial values $(p<0.05)$. Wilcoxon rank-sum test applied.

\section{Conclusion}

The methods of therapeutic angiogenesis related to liver cirrhosis imply using the exogenous factors and inducing the production of endogenous ones for the purpose of stimulating revascularization of microcirculatory bloodstream in the pathologically changed liver. This makes it possible not only to sustain its function due to an improvement in microcirculation and supplying hepatocytes with nutrients and oxygen, but also to reduce hepatic vascular resistance and, as a consequence, to decrease portal hypertension. ${ }^{10}$ Four the clinical studies used marrow and blood cells isolated on the basis of expression of the cell surface molecule CD34. ${ }^{11-14}$ This population includes hematopoietic stem and progenitor cells ${ }^{15}$ and endothelial progenitor cells. ${ }^{16}$ Endothelial progenitor cells have been shown to improve survival and hepatic function in animal models of liver disease. ${ }^{17-19}$ It is likely that the endothelial progenitor cells act, at least in part, by promoting new vessel formation and improving hepatocyte perfusion. It is known that the result of exposing tissue to high-intensity laser radiation is shock wave-induced barotrauma and thermal injury of the channels edges. Due to local hypoxia the 
size-limited burn scars cause neoangiogenesis, and the necrotically changed channel walls act as a morphological guiding structure for the stimulation of new vessels growth. ${ }^{20}$

When the cirrhotic liver on rat model is exposed to high-intensity laser radiation ( $\lambda-1064$ and $805 \mathrm{~nm})$, the cascade of consecutive reactions of satellite cells is caused by both the immediate effects, such as fat cells degranulation, the activation of thrombocytes with aggregates formation and $\alpha$ granules ejection, and by the marked macrophage infiltration, an increase in the number of fat cells, and the proliferation and higher synthetic activity of fibroblasts, which happens in the process of inflammation. This is accompanied by a local increase in the expression of basic fibroblasts growth factor, vascular endothelial growth factor, higher activity of matrix metalloproteinases and proteases of the plasmin system, which creates the necessary conditions for the proliferation and migration of endothelial and smooth muscle cells of the vessel wall, revascularization and the remodelling of tissue in the zones exposed to laser radiation. Moreover, the area of the newly-formed vessels remains at the initially reached level for at least 90 days. This is proved by the laser Doppler measurement of blood flow, showing that there is a statistically accurate increase in the flux motion index within the same period, which signifies that the microcirculation in the organ is restored to normal. ${ }^{21}$ The our clinical study revealed a beneficial effect of laser revascularization of the liver on portal hemodynamics in cirrhotic patients, which manifested itself as a reduction in the splanchnic venous congestion (decrease in diameter of the portal vein, flow volume of the portal vein, flow volume of the splenic vein, and consequently congestion index of the portal vein velocity), when Doppler sonography was carried out a year after the surgery. Therefore, improving of the microenvironment within the liver through formation of new vessels and extracellular matrix remodelling using a high-intensity laser radiation may be a promising method of treating both liver cirrhosis and its complications. Further validation of the results will be achieved through long-term follow-up of the patients and a larger number of studied subjects.

\section{Acknowledgements}

None.

\section{Conflicts of interest}

The authors declare that there is no conflict of interest.

\section{References}

1. Garbuzenko DV. Morphofunctional rearrangement of the hepatic vasculature in the pathogenesis of portal hypertension in liver cirrhosis. Ter Arkh. 2014;86(2):90-95.

2. Garbuzenko DV. The mechanisms of adaptation of the vascular bed to hemodynamic changes in portal hypertension. Vestn Ross Akad Med Nauk. 2013;(1):52-57.

3. Golovneva ES. Expression of basic fibroblast growth factor in the course of neoangiogenesis stimulated by high-intensity laser irradiation. Bull Exp Biol Med. 2002;134(1):95-97.

4. Golovneva ES, Popov GK. Expression of vascular endothelial growth factor during neoangiogenesis stimulated by exposure to high-intensity laser radiation. Bull Exp Biol Med. 2003;136(6):551-553.
5. Kovalenko VL, Abramovskaya NV, Garbuzenko DV. Morphological characterization of compensatory and adaptational response in the cirrhotic liver after exposure to high-intensity lazer radiation. Ural Med J. 2007;(12):75-78.

6. Baik SK. Haemodynamic evaluation by Doppler ultrasonography in patients with portal hypertension: a review. Liver Int. 2010;30(10):14031413.

7. Tsubaki T, Sato S, Fujikawa H, et al. Values of Doppler sonography predicts high risk variceal bleeding in patients with viral cirrhosis. Hepatogastroenterology. 2007;54(73):96-99.

8. Tasu JP, Rocher L, Peletier G, et al. Hepatic venous pressure gradients measured by duplex ultrasound. Clin Radiol. 2002;57(8):746-752.

9. Garbuzenko DV. Multiorgan hemodynamic disorder, conditionned by liver cirrhosis. Ter Arkh. 2007;79(2):73-77.

10. Garbuzenko DV. Mechanisms of compensation of structure and function of the liver at its damage and their practical significance. Roszhurn gastrojenterol gepatol koloproktol. 2008;17(6):14-21.

11. Mohamadnejad M, Namiri M, Bagheri M, et al. Phase 1 human trial of autologous bone marrow-hematopoietic stem cell transplantation in patients with decompensated cirrhosis. World $J$ Gastroenterol. 2007;13(24):3359-3363.

12. Khan AA, Parveen N, Mahaboob VS, et al. Safety and efficacy of autologous bone marrow stem cell transplantation through hepatic artery for the treatment of chronic liver failure: a preliminary study. Transplant Proc. 2008;40(4):1140-1144.

13. Levicar N, Pai M, Habib NA, et al. Long-term clinical results of autologous infusion of mobilized adult bone marrow derived CD34+ cells in patients with chronic liver disease. Cell Prolif. 2008;41(Suppl 1):115-125.

14. Pai M, Zacharoulis D, Milicevic MN, et al. Autologous infusion of expanded mobilized adult bone marrow-derived CD34+ cells into patients with alcoholic liver cirrhosis. Am $J$ Gastroenterol. 2008;103(8):1952-1958.

15. Colter M, Jones M, Heimfeld S. CD34+ progenitor cell selection: clinical transplantation, tumor cell purging, gene therapy, ex vivo expansion, and cord blood processing. J Hematother. 1996;5(2):179-184.

16. Asahara T, Murohara T, Sullivan A, et al. Isolation of putative progenitor endothelial cells for angiogenesis. Science. 1997;275(5302):964-967.

17. Taniguchi E, Kin M, Torimura T, et al. Endothelial progenitor cell transplantation improves the survival following liver injury in mice. Gastroenterology. 2006;130(2):521-531.

18. Ueno T, Nakamura T, Torimura T,et al. Angiogenic cell therapy for hepatic fibrosis. Med Mol Morphol. 2006;39(1):16-21.

19. Nakamura T, Torimura T, Sakamoto M, et al. Significance and therapeutic potential of endothelial progenitor cell transplantation in a cirrhotic liver rat model. Gastroenterology. 2007;133(1):91-107.

20. Muller GJ, Schaldach B. The effect of the laser on the tissue at transmyocardial revascularization. Laser medicine. 1998;2(1):4-8.

21. Golovneva ES, Kozel' AI, Popov GK. Pathophysiologic mechanisms of tissue revascularization using high intensity laser exposure. Vestn Ross Akad MedNauk. 2003;(6):23-26. 